\title{
Applications of the FDA's Counterfeit Detection Device (CD3+) to the Examination of Suspect Counterfeit Pharmaceutical Tablets and Packaging
}

\author{
S. Frank Platek ${ }^{1}$, Nicola Ranieri ${ }^{1}$ and JaCinta Batson ${ }^{1}$ \\ 1. US Food and Drug Administration - Forensic Chemistry Center, Cincinnati, Ohio, USA.
}

A primary area of research and case analyses performed at the US Food and Drug Administration's Forensic Chemistry Center (FCC) is the identification of counterfeit pharmaceutical tablets. In 2005, a concept was initiated to develop a device that would use alternative light sources enabling rapid examination and screening of suspect pharmaceutical tablets, relying upon differences in tablet component and coating fluorescence properties. The resultant device was named the Counterfeit Detection $\left(\mathrm{CD}^{*}\right)$ device, where “*” represents the version. Beginning with the CD1 which employed four selected visible light frequencies, each subsequent version has added frequency-specific light emitting diodes (LED's) and additional analytical capabilities. The current version, CD3+, carries an impressive array of LED's covering a wide range of frequencies from the ultraviolet and visible wavelengths (UV-VIS) and into the infrared (IR) region. (FIG 1) Other additions to the newer devices include a short wave UV source, an anti-Stokes source, an electro-magnetic field frequency detector, a digital handheld optical microscope, oscillating LED light sources (for hologram imaging), and tungsten for rich infrared source (for document examination). The CD3+ is currently used in a number of International mail and parcel import facilities by FDA investigators and scientists as well as other regulatory agencies. In addition, the CDx devices have been used in joint investigations with the US Customs and Border Patrol and internationally with the U.S. Center for Disease Control and Prevention and U.S. Pharmacopeial Convention in the investigation of counterfeit anti-malarial drugs in Asia [1] and Africa [2].

The use of the CDx devices has been overwhelmingly successful in the examination of suspect counterfeit pharmaceutical tablets and capsules. The newer versions of the device are now equipped with UV-VIS and IR CCD cameras. Selected camera lens filters permit a wide range from 255 $\mathrm{nm}$ to $\sim 1200 \mathrm{~nm}$ of fluorescence and absorbance imaging of suspect counterfeit tablets, with direct comparison to authentic tablets, or stored images of authentic tablets in a digital library on the device. (FIG 2) Field examination permits image capture of the suspect tablets, which may be stored for case processing or forwarded to the FCC for confirmation of a possible counterfeit. The decision may then be made to collect suspect items that are forwarded to the laboratory for analytical confirmation.

The CD3 and CD3+ devices also provide a method to examine the printing processes found on suspect pharmaceutical packaging including cartons, bottle labels, package inserts and outserts, syringe barrel labels, blister packs and various types of packaging material. The processes used to print packaging graphics (artwork) and variable data (lot and batch number, expiration date, etc.) are determined microscopically using a digital camera with its own LED illumination source. (FIG 3) The resultant images of tablets or packaging may be captured, stored and visually compared to authentic product packaging images also stored in the CDx library.

The use of the CDx devices has proven to be most valuable as a hand-held instrument for the rapid screening of large lots of suspect pharmaceutical samples. It provides a non-destructive initial screening of tablets, capsules and packaging, permitting identification of non-characteristic items which may be 
collected for further analyses to confirm authenticity. The library storage of tablet, capsule and packaging images is dynamic and increases with each investigation of new products. These features as well as others not discussed in the paper make the CDx device one of the most versatile and valuable anti-counterfeit pharmaceutical detection instruments available.

\section{References:}

[1] Nicola Ranieri et al, Am.J.Trop.Med.Hyg., 91(5), 2014, pp.920-924.

[2] Michael D. Green et al, Am.J.Trop.Med.Hyg., 92 (Suppl6), 2015, pp.8-16.
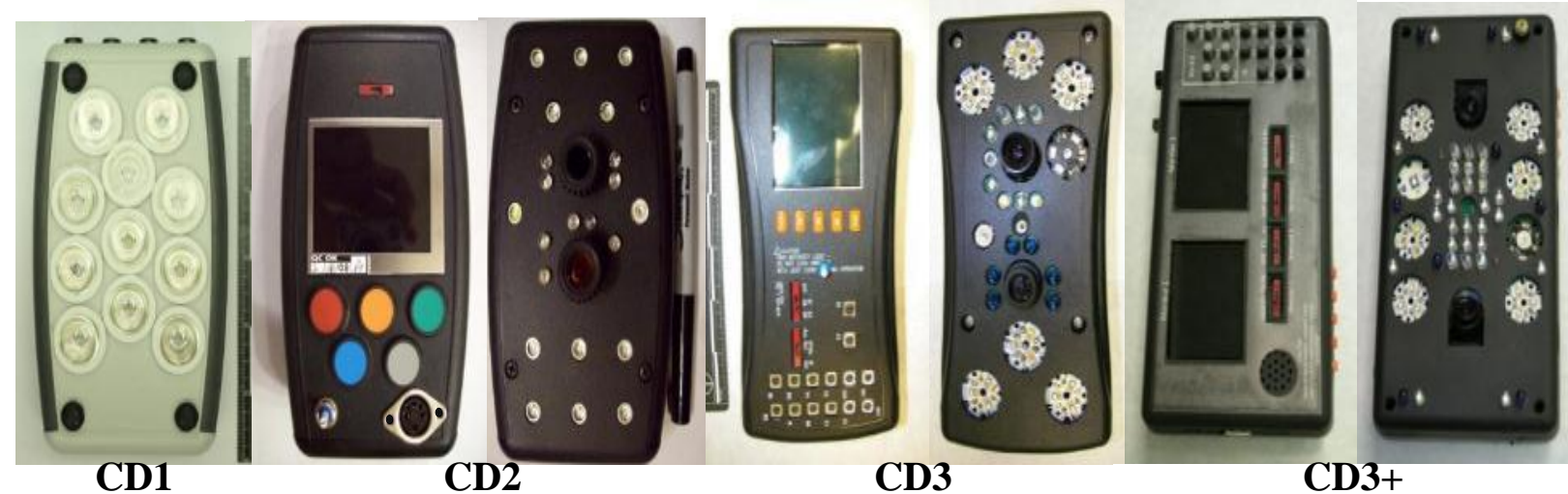

Figure 1. (L to R) The evolution of the $\mathrm{CD}$ devices from the $\mathrm{CD} 1$ to the $\mathrm{CD} 3+$.

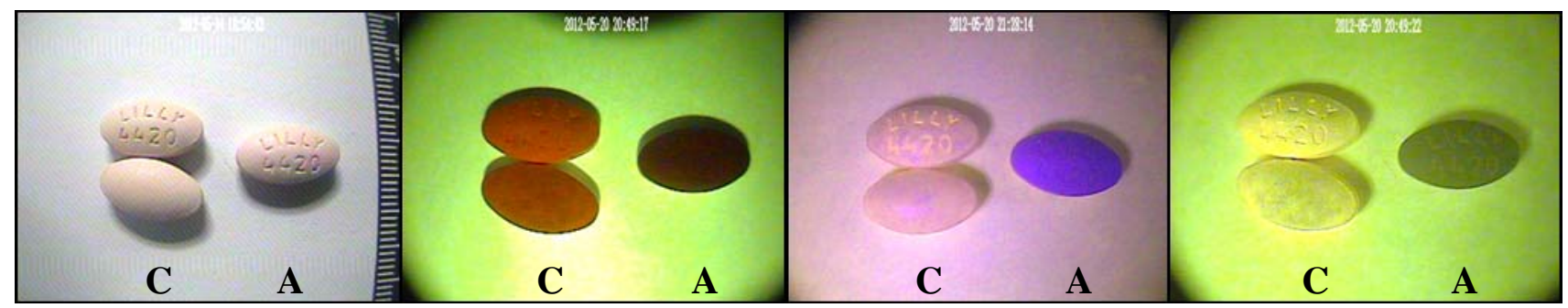

Figure 2. Imaging of counterfeit vs. authentic tablets using the CD3+ with white LED lights and three selected frequencies and filter combinations. $\mathrm{C}=$ counterfeit; $\mathrm{A}=$ Authentic

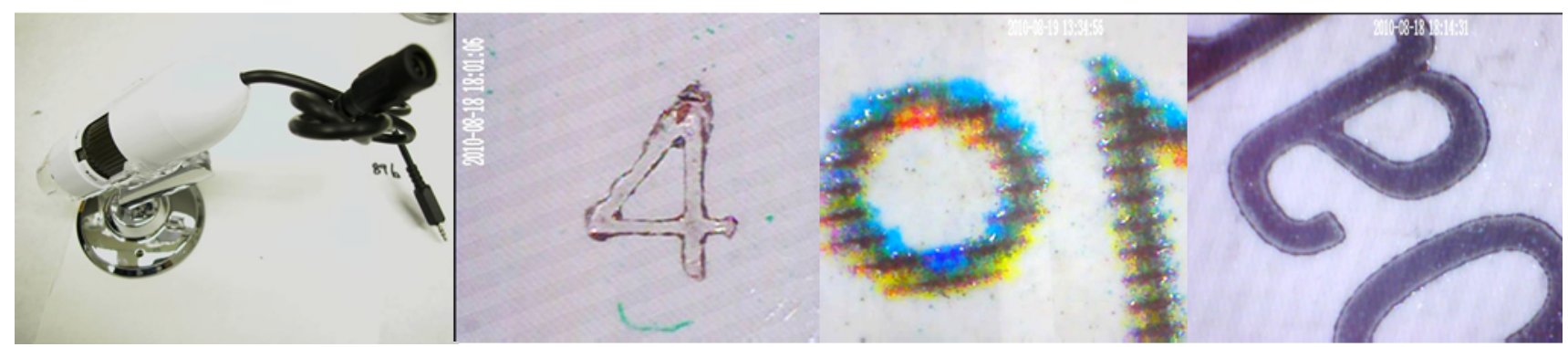

Figure 3. (L to R) Digital light microscope incorporated into the CD3+ showing examples of letterpress, electrographic and flexographic printing processes on pharmaceutical packaging. 